\title{
Singular Optimal Control of a 1-D Parabolic-Hyperbolic Degenerate Equation
}

\author{
Mamadou Gueye*, Pierre Lissy ${ }^{\dagger}$
}

\begin{abstract}
In this paper, we consider the controllability of a strongly degenerate parabolic equation with a degenerate one-order transport term. Despite the strong degeneracy, we prove a result of well-posedness and null controllability with a Dirichlet boundary control that acts on the degenerate part of the boundary. Then, we study the uniform controllability in the vanishing viscosity limit and prove that the cost of the control explodes exponentially fast in small time and converges exponentially fast in large time in some adapted weighted norm. The main tools used are a spectral decomposition involving Bessel functions and their zeros, some usual results on admissibility of scalar controls for diagonal semigroups, and the moment method of Fattorini and Russell.
\end{abstract}

Keywords: degenerate parabolic equations, cost of the control, uniform controllability, degenerate transport equation.

\section{Introduction}

Let $(\varepsilon, T, L, M, \alpha) \in(0,+\infty)^{4} \times(0,1)$. We consider the following degenerate controlled transport-diffusion equation :

$$
\begin{cases}y_{t}-\varepsilon\left(x^{\alpha+1} y_{x}\right)_{x}+M x^{\alpha} y_{x}=0 & \text { in }(0, L) \times(0, T), \\ y(0, t)=u(t), y(L, t)=0 & \text { on }(0, T), \\ y(x, 0)=y^{0}(x) & \text { in }(0, L) .\end{cases}
$$

The goal of this work is twofold :

(a) At fixed $(\varepsilon, T, L, M, \alpha) \in(0,+\infty)^{4} \times(0,1)$, we prove the well-posedness of (1) in appropriate interpolation spaces under a structural condition on these parameters. This provides an example of strongly degenerate parabolic equation with non-homogeneous Dirichlet boundary conditions.

(b) We prove the null controllability of (1), and letting $\varepsilon \rightarrow 0^{+}$, we perform an asymptotic analysis of the cost needed to control (1). Motivation for studying such singular optimal control emerges from the field of conservation laws in the context of weak entropy solutions.

Let us explain with more details the last point. Let $H$ be a Hilbert space, that will be described precisely later on, so that system (1) admits a unique solution for initial conditions in $H$. We say that system (1) is null controllable in $H$ at time $T$ with controls in $L^{2}(0, T)$ if for any $y^{0} \in H$ there exists $u \in L^{2}(0, T)$ such that $y(\cdot, T) \equiv 0$. Assume that (1) is null controllable in some space $H$, we denote by $U\left(\varepsilon, T, L, M, \alpha, y^{0}\right)$ the set of controls $u \in L^{2}(0, T)$ such that the corresponding solution of (1) satisfies $y(\cdot, T) \equiv 0$. Now, we can define the quantity which measures the cost of the null controllability for the system (1) as follows:

$$
\mathcal{K}(\varepsilon, T, L, M, \alpha):=\sup _{\left\|y^{0}\right\|_{H} \leq 1}\left\{\inf \left\{\|u\|_{L^{2}(0, T)}: u \in U\left(\varepsilon, T, L, M, \alpha, y^{0}\right)\right\}\right\} .
$$

*gueye.mamadou@gmail.com, Departamento de Matematica, Universidad Tecnica Federico Santa Maria, Casilla 110-V, Valparaiso, Chile. The author is partially supported by Fondecyt project 3140059.

† lissy@ceremade.dauphine.fr, CEREMADE, UMR 7534, Université Paris-Dauphine \& CNRS, Place du Maréchal de Lattre de Tassigny, 75775 Paris Cedex 16, France. The author is partially supported by the project ANR IFSMACS financed by the french Agence Nationale pour la Recherche. 
We are interested in the dependence of this cost with respect to $\varepsilon, T, L, M$ and $\alpha$ in the vanishing viscosity limit $\varepsilon \rightarrow 0^{+}$. In particular it is interesting to know whether or not this quantity remains bounded as $\varepsilon \rightarrow 0^{+}$. If it is the case, we will say that the system is uniformly null controllable.

To have an insight of how the cost $\mathcal{K}(\varepsilon, T, L, M, \alpha)$ behaves, it is natural to look at the limit system when $\varepsilon \rightarrow 0^{+}$:

$$
\begin{cases}y_{t}+M x^{\alpha} y_{x}=0 & \text { in }(0, L) \times(0, T), \\ y(0, t)=u(t) & \text { on }(0, T), \\ y(x, 0)=y^{0}(x) & \text { in }(0, L) .\end{cases}
$$

Let us point out that, as for system (1), it is not clear how to define the potential solutions of (3). The fact that the transport coefficient vanishes at $\{x=0\}$ and is not Lipschitz excludes the use of the standard theory of transport equations. Nevertheless, as for transport equations with BV coefficients (see [8] and [2]), it remains possible to prove the existence of solutions in a satisfactory sense. We postpone to Appendix B a definition and the proof of the existence and uniqueness of a solution to (3) in appropriate spaces, thanks to the method of characteristics.

By regarding the characteristics of (3) (see (100)), it is straightforward to observe that system (3) is null controllable if and only if $T \leqslant L^{1-\alpha} /((1-\alpha) M)$. Hence, we may expect that, as $\varepsilon \rightarrow 0^{+}$:

- $\mathcal{K}(\varepsilon, T, L, M, \alpha) \rightarrow \infty$ for $T<L^{1-\alpha} /((1-\alpha) M)$,

- $\mathcal{K}(\varepsilon, T, L, M, \alpha) \rightarrow 0$ for $T>L^{1-\alpha} /((1-\alpha) M)$.

However, proving this kind of result is very difficult even in the non-degenerate case (and might even be false, $c f[7])$. Our first main result, which concerns the uniform controllability of system (1), is the following.

Theorem 1.1. For every $(\varepsilon, T, L, M, \alpha) \in(0,+\infty)^{4} \times(0,1)$ such that $M / \varepsilon>\alpha$ and any $y^{0} \in$ $L^{2}\left((0,1) ; x^{-M / \varepsilon} d x\right)$, there exists a control $u \in L^{2}(0, T)$ such that the associated solution to (1) satisfies $y(\cdot, T) \equiv 0$. Moreover, there exists some constant $\mathcal{Q}(T, L, M, \alpha)>0$ such that, for every $(\varepsilon, T, L, M, \alpha) \in(0,+\infty)^{4} \times(0,1)$ with $M / \varepsilon>\alpha$ and $T$ verifying

$$
T>\frac{(2 \sqrt{6}) L^{1-\alpha}}{M(1-\alpha)},
$$

we have

$$
\mathcal{K}(\varepsilon, \alpha, T, L, M) \leq \exp \left(-\frac{\mathcal{Q}(T, L, M, \alpha)}{\varepsilon}\right) .
$$

Remark 1.1. Let us remark that this case covers a wide range of initial conditions, notably every initial condition with compact support or even every initial condition that vanishes faster than any polynomial, i.e. in $\bigcap_{\varepsilon \in(0, M / \alpha)} L^{2}\left((0,1) ; x^{-M / \varepsilon} d x\right)$.

The second main result of this work concerns a lower bound for the cost of controllability in small time.

Theorem 1.2. There exists some constant $\mathcal{R}(T, L, M, \alpha)>0$ such that, for every $(\varepsilon, T, L, M, \alpha) \in$ $(0,+\infty)^{4} \times(0,1)$ with $M / \varepsilon>\alpha$, for $\epsilon$ small enough and $T$ verifying

$$
T<\frac{(0,98) L^{1-\alpha}}{M(1-\alpha)},
$$

we have

$$
\mathcal{K}(\varepsilon, \alpha, T, L, M) \geq \exp \left(\frac{\mathcal{R}(T, L, M, \alpha)}{\varepsilon}\right) .
$$

Null controllability of uniformly parabolic equations in one dimension is by now well understood. In the case where the control is distributed or is acting on a part of the boundary we refer to $[9,10,18]$. The case of parabolic equations degenerating at the boundary of the domain have been tackled more recently, using similar methods with new ingredients. The case of distributed controls or controls acting on a part of the boundary where the equation is non-degenerate, solved 
in [4], is approached using global Carleman estimates (cf. [10]). When the control acts on a part of the boundary where degeneracy occurs, approximate null controllability was first obtained in [5] using local Carleman estimates ( $c f$. [18]). Then, this last result has been extended to exact null controllability in [15], using the moment method (cf. [9]) together with the transmutation method.

Let us now give a quick overview of some results concerning the study of the uniform controllability in singular limits. For more details concerning the exact scope of the existing results, we refer to the introduction of [24]. The first example of study was given in [7], where the authors studied the case of a transport-diffusion equation with constant coefficients in the vanishing viscosity limit, with positive or negative speed of propagation. In this paper, the authors proved that the cost of the control has to explode in small time and decreases exponentially in large time, however there is still a range of times for which the exact behavior of the cost of the control is unknown. The study of this simpler case has been continued in [11, 21, 22] (in these two last articles, a link is notably made between the vanishing viscosity problem and another famous problem concerning the cost of fast controls for the heat equation) and [24]. Let us also mention some extensions for varying in time and space speed $M$ in arbitrary space dimension in [14], for conservation laws in [20] and for vanishing dispersion in $[12,13,6]$.

The rest of the paper is organized as follows:

- In section 2.1, we give some notations and set our functional framework.

- In section 2.2, we perform a careful spectral analysis of the degenerate elliptic operator appearing in (1). As usual for degenerate operators, the eigenfunctions will be expressed thanks to Bessel functions and the eigenvalues thanks to the zeros of these Bessel functions.

- In section 2.3, we give a well-posedness result for system (1) in appropriate spaces. The main tools used are the previous spectral decomposition and a result concerning the admissibility of scalar control operators for diagonal semigroups proved in [17].

- In section 3.1, we prove our first Theorem concerning the null controllability and the exponential decreasing of the cost of the control in large time when $\varepsilon \rightarrow 0^{+}$. We use the moment method (see [9]), which requires to study carefully some infinite product involving the eigenvalues and to exhibit an appropriate multiplier, which will be the one used in [32].

- To conclude, in section 3.2, we prove our second Theorem concerning the explosion of the cost of controllability in small time when $\varepsilon \rightarrow 0^{+}$. The main ingredient of the proof is to consider some "quasi-optimal control" bringing the first eigenfunction of the elliptic operator to 0 at time $T$ and the use of tools coming from complex analysis (notably an appropriate representation Theorem for entire functions) in the spirit of [7].

\section{Preliminaries}

\subsection{Functional setting}

Here we give some notations and introduce some necessary materials.

We introduce an unbounded operator $\mathcal{A}: D(\mathcal{A}) \subset L^{2}\left((0, L) ; x^{-M / \varepsilon} \mathrm{d} x\right) \rightarrow L^{2}\left((0, L) ; x^{-M / \varepsilon} \mathrm{d} x\right)$ given by

$$
\left\{\begin{array}{l}
D(\mathcal{A}):=\left\{y \in D_{\max }(\mathcal{A}) \mid \lim _{x \rightarrow 0, L} y(x)=0\right\} \\
\forall y \in D(\mathcal{A}), \mathcal{A}[y](x):=-\varepsilon\left(x^{\alpha+1} y_{x}\right)_{x}+M x^{\alpha} y_{x}
\end{array}\right.
$$

where

$$
D_{\max }(\mathcal{A})=\left\{u \in L^{2}\left((0, L) ; x^{-M / \varepsilon} \mathrm{d} x\right): u, u^{\prime} \in A C_{\mathrm{loc}}, \mathcal{A} u \in L^{2}\left((0, L) ; x^{-M / \varepsilon} \mathrm{d} x\right)\right\} .
$$

Then, under the structural assumption $M / \varepsilon>\alpha$, it is proven that $(\mathcal{A}, D(\mathcal{A}))$ is self-adjoint on $L^{2}\left((0, L) ; x^{-M / \varepsilon} \mathrm{d} x\right)$ and generates an analytic semigroup of bounded linear operators $S(t)$ (see $\left[29\right.$, section 4]). Besides, there exists a Hilbert basis $\left(\Phi_{n}\right)_{n \in \mathbb{N} \backslash\{0\}}$ of $L^{2}\left((0, L) ; x^{-M / \varepsilon} \mathrm{d} x\right)$ and a sequence $\left(\lambda_{n}\right)_{n \in \mathbb{N} \backslash\{0\}}$ of real numbers, with $\lambda_{n}>0$ and $\lambda_{n} \rightarrow \infty$, such that

$$
\mathcal{A} \Phi^{n}=\lambda_{n} \Phi^{n} \quad \forall n \in \mathbb{N} \backslash\{0\} .
$$


Now, thanks to the continuous functional calculus, we can introduce appropriate interpolation spaces for the initial data [31, pp 35-39]. For any $s \geq 0$, we define

$$
\mathcal{H}^{s}(0, L):=\left\{u=\sum_{n \in \mathbb{N} \backslash\{0\}} a_{n} \Phi^{n} /\|u\|_{\mathcal{H}^{s}}^{2}:=\sum_{n \in \mathbb{N} \backslash\{0\}}\left|a_{n}\right|^{2} \lambda_{n}^{s}<\infty\right\},
$$

and let

$$
\mathcal{H}^{-s}(0, L):=\left[\mathcal{H}^{s}(0, L)\right]^{\prime}
$$

We denote the duality product by $\langle\cdot, \cdot\rangle_{\mathcal{H}^{-s}, \mathcal{H}^{s}}$, since the spacial domain will not change. Let us recall that for $f:=\sum a_{n} \Phi^{n} \in \mathcal{H}^{-s}$ and $g:=\sum b_{n} \Phi^{n} \in \mathcal{H}^{s}$, it is easy to compute the duality product $\langle f, g\rangle_{\mathcal{H}^{-s}, \mathcal{H}^{s}}$ by the following formula:

$$
\langle f, g\rangle_{\mathcal{H}^{-s}, \mathcal{H}^{s}}=\sum_{n \in \mathbb{N} \backslash\{0\}} a_{n} b_{n} .
$$

for a time $T>0$ and a real number $\ell$ we let $H^{\ell}(0, T)$ be the usual Sobolev space and we will use Bochner type spaces $H^{\ell}\left(0, T ; \mathcal{H}^{s}\right)$ (see [26, page 11]).

For any $z \in D_{\max }(\mathcal{A})$ we denote by $\mathcal{O}_{\varepsilon, M, \alpha}(z)$ the generalized derivative at $\{x=0\}$, that is

$$
\mathcal{O}_{\varepsilon, M, \alpha}(z):=\lim _{x \rightarrow 0} x^{\alpha+1-M / \varepsilon} z^{\prime}(x) .
$$

\subsection{Spectral analysis}

We aim at giving explicit expression of the eigenfunctions and eigenvalues of the spectral problem

$$
\left\{\begin{array}{l}
\mathcal{A}[\varphi](x)=\lambda \varphi(x), \quad x \in(0,1), \\
\varphi(0)=\varphi(1)=0,
\end{array}\right.
$$

where $\mathcal{A}$ was defined in (8). This is a special case of Sturm-Liouville problem. Indeed, let us consider the following general case of Bessel equation

$$
x^{2} y^{\prime \prime}+a x y^{\prime}+\left(b x^{\ell}+c\right) y=0, \quad x \in(0, \infty),
$$

where $a, b$, and $c$ are real numbers and $\ell \neq 0$. Then, referring to [16, Section C, Equation 2.162(1a)] the solutions of (15) can be written as follows:

$$
\begin{cases}b \neq 0: & y(x)=x^{\frac{1}{2}(1-a)} Z_{\nu}\left(\kappa^{-1} \sqrt{b} x^{\kappa}\right), \\
b=0: & y(x)=\left\{\begin{array}{l}
C_{1} x^{\frac{1}{2}(1-a+\ell \nu)}+C_{2} x^{\frac{1}{2}(1-a-\ell \nu)} \text { if } \quad \nu \neq 0, \\
x^{\frac{1}{2}(1-a)}\left(C_{1}+C_{2} \log x\right) \quad \text { if } \quad \nu=0,
\end{array}\right.\end{cases}
$$

where

$$
\nu:=\frac{1}{\ell} \sqrt{(1-a)^{2}-4 c}, \quad \kappa:=\frac{\ell}{2},
$$

and $Z_{\nu}$ is any Bessel function of order $\nu$ (see Appendix A for some results concerning Bessel functions).

The Sturm-Liouville problem (14) is exactly (15) with

$$
a=\alpha+1-\frac{M}{\varepsilon}, \quad b=\frac{\lambda}{\varepsilon}, \quad \ell=1-\alpha, \quad c=0 .
$$

Then, from (16) we infer

$$
\varphi(x, \lambda)=x^{\frac{1}{2}\left(\frac{M}{\varepsilon}-\alpha\right)} Z_{\nu}\left(\kappa^{-1} \sqrt{\frac{\lambda}{\varepsilon}} x^{\kappa}\right), \quad x \in(0, \infty), \lambda \in \mathbb{R},
$$

with the real parameters $\nu$ and $\kappa$ defined by

$$
\nu:=(M / \varepsilon-\alpha) /(1-\alpha), \quad \kappa:=\frac{1}{2}(1-\alpha) .
$$


Now, assuming that $\varepsilon$ is small enough such that $M / \varepsilon-\alpha>0$, using the boundary conditions in (14) one gets

$$
\varphi\left(x, \lambda_{n}\right)=x^{\frac{1}{2}(M / \varepsilon-\alpha)} J_{\nu}\left(\kappa^{-1} \sqrt{\frac{\lambda_{n}}{\varepsilon}} x^{\kappa}\right), \quad x \in(0,1),
$$

where

$$
\lambda_{n}:=\varepsilon\left(\kappa j_{\nu, n}\right)^{2}, n \in \mathbb{N} \backslash\{0\} .
$$

Now, using Lemma A.3 we normalize our eigenvectors in the $L^{2}\left((0,1) ; x^{-M / \varepsilon} \mathrm{d} x\right)$-norm :

$$
\Phi^{n}(x):=\frac{(2 \kappa)^{\frac{1}{2}}}{\left|J_{\nu}^{\prime}\left(j_{\nu, n}\right)\right|} x^{\frac{1}{2}(M / \varepsilon-\alpha)} J_{\nu}\left(j_{\nu, n} x^{\kappa}\right), \quad x \in(0,1) .
$$

Let us prove that the family $\left\{\Phi^{n}, n \in \mathbb{N} \backslash\{0\}\right\}$ form an Hilbert basis of $L^{2}\left((0,1) ; x^{-M / \varepsilon} \mathrm{d} x\right)$ by checking the Bessel equality. Let $f \in L^{2}\left((0,1) ; x^{-M / \varepsilon} \mathrm{d} x\right)$ and let

$$
a_{n}:=\int_{0}^{1} f(x) \Phi^{n}(x) x^{-M / \varepsilon} \mathrm{d} x .
$$

Then, using Lemma A.3 and changing variables twice

$$
\begin{gathered}
\sum_{n \in \mathbb{N} \backslash\{0\}}\left|a_{n}\right|^{2}=\sum_{n \in \mathbb{N} \backslash\{0\}}\left|\int_{0}^{1} f(x) \frac{(2 \kappa)^{\frac{1}{2}}}{\left|J_{\nu}^{\prime}\left(j_{\nu, n}\right)\right|} x^{\frac{1}{2}\left(\frac{M}{\varepsilon}-\alpha\right)} J_{\nu}\left(j_{\nu, n} x^{\kappa}\right) x^{-M / \varepsilon} \mathrm{d} x\right|^{2} \\
=\kappa \sum_{n \in \mathbb{N} \backslash\{0\}}\left|\int_{0}^{1} \kappa^{-1} f\left(y^{1 / \kappa}\right) y^{-(M / \varepsilon-\alpha) / 2 \kappa+(1-\kappa) / \kappa} \frac{\sqrt{2 y}}{\left|J_{\nu}^{\prime}\left(j_{\nu, n}\right)\right|} J_{\nu}\left(j_{\nu, n} y\right) \mathrm{d} y\right|^{2} \\
=\int_{0}^{1} \kappa^{-1}\left|f\left(y^{1 / \kappa}\right)\right|^{2} y^{-(M / \varepsilon-\alpha) / \kappa+2(1-\kappa) / \kappa} \mathrm{d} y=\int_{0}^{1}|f(x)|^{2} x^{-M / \varepsilon} \mathrm{d} x
\end{gathered}
$$

Remark 2.1. One can observe that it is crucial in our spectral analysis that $M / \varepsilon>\alpha$, and the computations above would not work in the case $M \leqslant 0$. This explains why we are able here to define boundary Dirichlet conditions at the boundary, contrary to the case where $M=0$.

We finish by giving a formula for the generalized derivatives of our eigenfunctions at the degeneracy point.

Lemma 2.1. We have the following equalities:

$$
\lim _{x \rightarrow 0} x^{\alpha+1-M / \varepsilon} \Phi_{x}^{n}(x):=\mathcal{O}_{\varepsilon, M, \alpha}\left(\Phi^{n}\right)=\frac{(M / \varepsilon-\alpha)(2 \kappa)^{1 / 2}\left(j_{\nu, n}\right)^{\nu}}{2^{\nu}\left|J_{\nu}^{\prime}\left(j_{\nu, n}\right)\right| \Gamma(\nu+1)} \quad \forall n \in \mathbb{N} \backslash\{0\} .
$$

The proof of this Lemma is straightforward using (86) and the recurrence formula (87).

\subsection{Well-posedness of the boundary control problem (1)}

In this section we make precise what we mean by a weak solution of (1). The functional setting and the well-posedness are crucial issues in this work. This result could be seen as an immediate consequence of the admissibility results for diagonal operators and scalar input control given in [17]. One has to be careful that in [17], the authors consider $\mathcal{H}^{-s}$ itself as the pivot space (which is not our case here since our pivot space is $L^{2}\left((0, L), x^{-M / \varepsilon}\right)$ ) and then the equivalent of $(25)$ is written using the inner product $\langle\cdot, \cdot\rangle_{\mathcal{H}^{-s}}$. Here, we decided to work with the duality product $\langle\cdot, \cdot\rangle_{\mathcal{H}^{-s}, \mathcal{H}^{s}}$ (see [26]), however one easily verifies that Theorem 2.2 of [17] can be easily adapted to this case.

Definition 2.1. Let $(\varepsilon, T, L, M, \alpha) \in(0,+\infty)^{2} \times[0,1)$ such that $M / \varepsilon-\alpha>0$, let $y^{0} \in \mathcal{H}^{-s}$, for some $s>0$ and $u \in L^{2}(0, T)$. A weak solution of $(1)$ is a function $y \in \mathcal{C}^{0}\left([0, T] ; \mathcal{H}^{-s}\right)$ such that for every $\tau \in(0, T]$ and for every $z^{\tau} \in \mathcal{H}^{s}$ one has

$$
\left\langle y(\tau), z^{\tau}\right\rangle_{\mathcal{H}^{-s}, \mathcal{H}^{s}}=\int_{0}^{\tau} u(t) \mathcal{O}_{\varepsilon, M, \alpha}\left(S(\tau-t) z^{\tau}\right) d t+\left\langle y^{0}, z(0)\right\rangle_{\mathcal{H}^{-s}, \mathcal{H}^{s}}
$$


Let us give a result of well-posedness for this problem.

Proposition 2.1. Let $(\varepsilon, T, L, M, \alpha) \in(0,+\infty)^{4} \times[0,1)$ such that $M / \varepsilon>\alpha, u \in L^{2}(0, T)$ and $y^{0} \in \mathcal{H}^{-s}$, such that $s \geq \nu-1 / 2$ where $\nu=(M / \varepsilon-\alpha) /(1-\alpha)$. Then, the formula (25) defines, for each $\tau \in[0, T]$, a unique element $y(\tau) \in \mathcal{H}^{-s}$ that can be written as

$$
y(\tau)=S(\tau) y^{0}+B(\tau) u, \quad \tau \in(0, T],
$$

Where $B(\tau)$ is the strongly continuous family of bounded operators $B(\tau): L^{2}(0, T) \rightarrow \mathcal{H}^{-s}$ given by

$$
\left\langle B(\tau) u, z^{\tau}\right\rangle_{\mathcal{H}^{-s}, \mathcal{H}^{s}}=\int_{0}^{\tau} u(t) \mathcal{O}_{\varepsilon, M, \alpha}\left(S(\tau-t) z^{\tau}\right) d t, \quad \forall z^{\tau} \in \mathcal{H}^{s}
$$

Moreover, the unique weak solution y on $[0, T]$ to $(1)$ (in the sense of $(25))$ belongs to $\mathcal{C}^{0}\left([0, T] ; \mathcal{H}^{-s}\right)$ and verifies

$$
\|y\|_{L^{\infty}\left([0, T] ; \mathcal{H}^{-s}\right)} \leq C\left(\left\|y^{0}\right\|_{\mathcal{H}^{-s}}+\|u\|_{L^{2}(0, T)}\right) .
$$

Proof of Proposition 2.1. Using the fact that for $s>0$,

$$
\mathcal{H}^{s} \subset L^{2}\left((0,1) ; x^{-M / \varepsilon} \mathrm{d} x\right) \subset \mathcal{H}^{-s},
$$

with dense embeddings, it is clear that

$$
y(\tau)-S(\tau) y^{0}=\zeta(\tau)
$$

where

$$
\left\langle\zeta(\tau), z^{\tau}\right\rangle_{\mathcal{H}^{-s}, \mathcal{H}^{s}}=\int_{0}^{\tau} u(t) \mathcal{O}_{\varepsilon, M, \alpha}\left(S(\tau-t) z^{\tau}\right) \mathrm{d} t, \quad \forall z^{\tau} \in \mathcal{H}^{s} .
$$

Then, it suffices to prove that the operator $\zeta(\tau)$ defined by (29) has a continuous extension to $\mathcal{H}^{-s} \rightarrow L^{2}(0, T)$. To do so, let $z^{\tau} \in \mathcal{H}^{s}$, such that $s>0$, that we expand as follows:

$$
z^{\tau}(x)=\sum_{n \in \mathbb{N} \backslash\{0\}} b_{n} \Phi^{n}(x) .
$$

We infer

$$
S(\tau-t) z^{\tau}(x)=\sum_{n \in \mathbb{N} \backslash\{0\}} \exp \left\{\lambda_{n}(t-\tau)\right\} b_{n} \Phi^{n}(x), \quad \forall t \in[0, \tau] .
$$

Using Lemma 2.1 and Lemma A.4, we obtain

$$
\begin{gathered}
\int_{0}^{\tau}\left|\mathcal{O}_{\varepsilon, M, \alpha}\left(S(\tau-t) z^{\tau}\right)\right|^{2} \mathrm{~d} t=\int_{0}^{\tau} \sum_{n \in \mathbb{N} \backslash\{0\}}\left|b_{n}\right|^{2}\left|\frac{(M / \varepsilon-\alpha)(2 \kappa)^{1 / 2}\left(j_{\nu, n}\right)^{\nu}}{2^{\nu} \Gamma(\nu+1)\left|J_{\nu}^{\prime}\left(j_{\nu, n}\right)\right|} \exp \left\{\lambda_{n}(t-\tau)\right\}\right|^{2} \mathrm{~d} t \\
\leq C\left\|z^{\tau}\right\|_{\mathcal{H}^{s}},
\end{gathered}
$$

as soon as $s \geq \nu-1 / 2$. Then, following the proof of [17, Theorem 2.2], we conclude the proof.

Remark 2.2. We will see in the following that from now on we essentially work with initial conditions belonging to $L^{2}\left((0, L), x^{-M / \varepsilon}\right)$. This is not a problem since $L^{2}\left((0, L), x^{-M / \varepsilon}\right) \subset \mathcal{H}^{-\nu+1 / 2}$, so the result of Proposition 2.1 applies with $s=\nu-1 / 2$, however one has to be careful that we will not have that the corresponding solution y belongs to $C^{0}\left([0, T], L^{2}\left((0, L), x^{-M / \varepsilon}\right)\right)$ but only to $C^{0}\left([0, T], \mathcal{H}^{-\nu+1 / 2}\right)$.

Remark 2.3. In all what follows, it is enough to consider the case $L=1$ and $M=1$. Indeed, this is a consequence of the following scaling argument: if $y$ is a solution of $(1)$, then $z(t, x):=$ $y\left(L^{1-\alpha} t / M, L x\right)$ is solution of

$$
\begin{cases}y_{t}-\tilde{\varepsilon}\left(x^{\alpha+1} y_{x}\right)_{x}+x^{\alpha} y_{x}=0 & \text { in }(0, \tilde{T}) \times(0,1) \\ y(0, t)=u(t), y(1, t)=0 & \text { on }(0, \tilde{T}) \\ y(x, 0)=y^{0}(x) & \text { in }(0,1)\end{cases}
$$

where $\tilde{T}:=T M / L^{1-\alpha}$ and $\tilde{\varepsilon}:=\varepsilon / M$. 


\section{Proof of the Theorems 1.1 and 1.2}

\subsection{Convergence of the cost of null controllability in large time}

During all the proof, we will consider $L=1$ and $M=1$ (see Remark 2.3). Moreover, for the sake of simplicity, we will denote by $F(\varepsilon, T, \alpha)$ some fractional function of $\varepsilon, T, \alpha$ that may vary from line to line (its behavior is negligible with respect to the exponential terms in $\varepsilon$ and $T$ that will appear).

We are going to proceed as in the seminal work [9], i.e. we will use the moment method (see also [3]) to solve the controllability problem. This method consists in finding a bi-orthogonal family $\left\{\Psi_{k}\right\}_{k \in \mathbb{N}^{*}}$ to the family of exponential $\left\{e^{-\lambda_{k}(T-t)}\right\}_{k \in \mathbb{N} \backslash\{0\}}$ on $[0, T]$, i.e. verifying

$$
\int_{0}^{T} \Psi_{k}(t) e^{-\lambda_{\ell}(T-t)} d t=\delta_{k \ell}, \quad k, \ell \in \mathbb{N}^{*} .
$$

This will enable us to provide an upper bound on the cost of the control. The strategy to construct such a bi-orthogonal sequence is based on the Paley-Wiener theorem. After some translation argument that we be given in details later, one can reduce the problem to finding a bi-orthogonal family $\left\{\bar{\Psi}_{k}\right\}_{k \in \mathbb{N} \backslash\{0\}}$ to $\left\{e^{\lambda_{k} t}\right\}_{k \in \mathbb{N} \backslash\{0\}}$ on $[-T / 2, T / 2]$.

For the time being, consider $J_{k}$ the Fourier transform of $\bar{\Psi}_{k}$. Then, $\left\{\bar{\Psi}_{k}\right\}_{k \in \mathbb{N} \backslash\{0\}}$ is biorthogonal to the family of exponentials $\left\{e^{\lambda_{k} t}\right\}_{k \in \mathbb{N} \backslash\{0\}}$ on $[-T / 2, T / 2]$ if and only if

$$
J_{k}\left(i \lambda_{\ell}\right)=\delta_{k \ell}, \quad k, \ell \in \mathbb{N} \backslash\{0\} .
$$

The first idea to exhibit such a family $\left\{J_{k}\right\}_{k \in \mathbb{N} \backslash\{0\}}$ could be to consider a single entire function having simple zeros at $\left\{i \varepsilon\left(\kappa j_{\nu, k}\right)^{2}, k \in \mathbb{N} \backslash\{0\}\right\}$. Thus, let us begin with exhibiting such an entire function. The function

$$
\Lambda(z):=\prod_{k=1}^{+\infty}\left(1+\frac{i z}{\varepsilon\left(\kappa j_{\nu, k}\right)^{2}}\right)
$$

has exactly its zeros at these points. Using [33, Chap. XV, Page 498, (3)], we know that

$$
\Lambda(z)=\Gamma(\nu+1)\left(\frac{2 \sqrt{\varepsilon} \kappa}{\sqrt{i z}}\right)^{\nu} J_{\nu}\left(-\frac{\sqrt{i z}}{\sqrt{\varepsilon} \kappa}\right) .
$$

Let us give some basic properties of this infinite product.

Lemma 3.1. 1. If $z \in \mathbb{C}$,

$$
|\Lambda(z)| \leq \exp \left(\frac{\sqrt{|z|}}{\kappa \sqrt{\varepsilon}}\right)
$$

2. If $x \in \mathbb{R}$,

$$
|\Lambda(x)| \leq \exp \left(\frac{\sqrt{|x|}}{\kappa \sqrt{2 \varepsilon}}\right) .
$$

Proof of Lemma 3.1: Using (35) and taking into account (85), we infer that

$$
|\Lambda(z)| \leqslant e^{\frac{|\Im(\sqrt{i z})|}{\sqrt{\varepsilon} \kappa}},
$$

from which we easily deduce the result, taking into account that $\sqrt{i}=(1+i) / 2$.

Once $\Lambda$ is given, and because the roots of $\Lambda$ are exactly the $i \lambda_{k}$ and are simple, the most natural choice for $J_{k}$ would be the following:

$$
\psi_{k}(z):=\frac{\Lambda(z)}{\Lambda^{\prime}\left(i \lambda_{k}\right)\left(z-i \lambda_{k}\right)}, \quad k \in \mathbb{N} \backslash\{0\} .
$$

However, since the asymptotic given in (37) is optimal at infinity, $\Phi_{k}$ cannot be in $L^{2}$ and it cannot be directly used to obtain a bi-orthogonal family in $L^{2}(0, T)$ (the Paley-Wiener theorem 
cannot be applied). Nevertheless, we will slightly modify these functions in order to make them bounded and still of exponential type without perturbing their zeros.

Let us now introduce the multiplier, that is very similar to the one used in [32]. Let $\theta>0$ and $\beta>0$ to be chosen later, and define

$$
\sigma_{\theta}(t):=\exp \left(-\frac{\theta}{1-t^{2}}\right)
$$

prolonged by 0 outside $(-1 ; 1)$, then $\sigma_{\theta}$ is analytic on $B(0,1)$. We call

$$
H_{\beta}(z):=C_{\theta} \int_{-1}^{1} \sigma_{\theta}(t) e^{-i \beta t z} d t
$$

where

$$
C_{\theta}:=\left(\int_{-1}^{1} \sigma_{\theta}(t) d t\right)^{-1}
$$

In the following, $F(\beta, \theta)$ will denote some fractional function of $\theta$ and $\beta$ that may vary from line to line. The following result gives the main properties needed for the multiplier.

Lemma 3.2. The function $H_{\beta}$ defined by (39) verifies the following inequalities:

$$
\begin{gathered}
H_{\beta}(i x) \geq F(\beta, \theta), \\
\left|H_{\beta}(z)\right| \leqslant F(\beta, \theta) e^{\beta|\Im(z)|}, \\
H_{\beta}(x) \leqslant F(\beta, \theta) \sqrt{|x|} \exp \left(\frac{3 \theta}{4}-\sqrt{\beta \theta x}\right) .
\end{gathered}
$$

Estimates (40) and (41) directly follow from [32, Page 85], whereas (42) can be easily deduced from the beginning of [32, Page 86].

We then introduce

$$
J_{k}(z):=\psi_{k}(z) \frac{H_{\beta}(z)}{H_{\beta}\left(i \lambda_{k}\right)} .
$$

Let us now explain how we choose $\theta$ and $\beta$. Let $\delta>0$ be a small parameter. We want to apply the Paley-Wiener Theorem on the interval $[-T / 2, T / 2]$, so that after some translation our control will have its support included in $[0, T]$. Hence, we have to choose $\beta$ close to $T / 2$ and we set $\beta$ as follows:

$$
\beta:=\frac{T(1-\delta)}{2} \text {. }
$$

Now, we want to "compensate" the bad growth of $\psi$ on the real line given by (37) thanks to the "good" decreasing of the multiplier given in (42), hence we have to choose $\sqrt{\beta \theta}$ close to $1 /(\kappa \sqrt{2 \varepsilon})$. Taking into account (44), we choose

$$
\theta:=\frac{1+\delta}{\varepsilon \kappa^{2} T(1-\delta)} .
$$

Let us give precise estimates on $J_{k}$.

Lemma 3.3. For any $k \in \mathbb{N} \backslash\{0\}$ the function $J_{k}$ is of exponential type $T / 2, J_{k} \in L^{1}(\mathbb{R}) \cap L^{2}(\mathbb{R})$ and verifies

$$
J_{k}\left(i \lambda_{l}\right)=\delta_{k l} \quad \forall k, \ell \in \mathbb{N} \backslash\{0\},
$$

moreover there exists a constant $C>0$ (independant of $\theta$ and $\beta$ ) such that

$$
\left\|J_{k}\right\|_{L^{1}(\mathbb{R})} \leqslant \frac{1}{\lambda_{k}\left|\Lambda^{\prime}\left(i \lambda_{k}\right)\right|} F(\varepsilon, T, \alpha) e^{\frac{3}{4 \theta}} .
$$

\section{Proof of Lemma 3.3.}

Using (41) together with (44), (36) and (43), we easily see that $J_{k}$ is of exponential type $T / 2$. Moreover, thanks to (43) and (38), $J_{k}$ verifies (46). Now, we want to estimate the $L^{1}-$ norm of $J_{k}$ on the real axis. Using $(43),(37),(40)$ at point $-\lambda_{k},(42),(44)$ and (45), we infer that

$$
\left|J_{k}(x)\right| \leqslant \frac{C}{\left|\Lambda^{\prime}\left(i \lambda_{k}\right)\left(x-i \lambda_{k}\right)\right|}(\theta+1) e^{3 \theta / 4-(1+\delta / 2) \sqrt{\frac{|x|}{2 \varepsilon}}} e^{\sqrt{\frac{|x|}{2 \varepsilon}}}
$$


We remark that (by using the change of variables $t=\frac{\delta}{2} \sqrt{\frac{x}{2 \varepsilon}}$ )

$$
\int_{\mathbb{R}} e^{-\frac{\delta}{2} \sqrt{\frac{|x|}{2 \varepsilon}}} d x \leqslant C \varepsilon
$$

Using (48) and (49), we deduce that $J_{k}(x) \in L^{1}(\mathbb{R}) \cap L^{2}(\mathbb{R})$ and that (47) holds.

We are now ready to prove Theorem 1.1.

\section{Proof of Theorem 1.1.}

Using Lemma 3.3, we deduce, by applying the version of the Paley-Wiener Theorem given in [30, Theorem 19.3, Page 370], that $J_{k}$ is the Fourier-Laplace transform of some function $f_{k} \in L^{2}(\mathbb{R})$ with compact support in $[-T / 2, T / 2]$. Let us consider

$$
g_{n}(t):=e^{T \lambda_{k} / 2} f_{k}(t-T / 2) .
$$

$g_{n}$ is supported in $[0, T]$ and is bi-orthogonal to the family $\left\{e^{-\lambda_{l}(T-t)}\right\}$ because thanks to (46) we have

$$
\int_{0}^{T} g_{k}(t) e^{-\lambda_{l}(T-t)} d t=\int_{0}^{T} f_{k}(t-T / 2) e^{T \lambda_{k} / 2} e^{-\lambda_{k}(T-t)} d t=\int_{-T / 2}^{T / 2} f_{k}(s) e^{T \lambda_{k} / 2} e^{-\lambda_{k}(T / 2-s)} d s=\delta_{k l} .
$$

We are now able to solve our moment problem. Let us consider some initial condition $y^{0} \in L^{2}\left(x^{-\frac{1}{\varepsilon}}\right)$, that we decompose in the orthonormal basis given by the $\Phi^{n}$ :

$$
y_{0}(x)=\sum_{n \in \mathbb{N} \backslash\{0\}} a_{n} \Phi^{n}(x) .
$$

We set

$$
u(t):=\sum_{n \in \mathbb{N} \backslash\{0\}}-\frac{a_{n} e^{-\lambda_{n} T}}{\mathcal{O}_{\varepsilon, M, \alpha}\left(\Phi^{n}\right)} g_{n}(t),
$$

provided that $1 / \varepsilon>\alpha$ (see expression (24)). Using (51) and (12), we infer that

$$
\int_{0}^{T} u(t) \mathcal{O}_{\varepsilon, M, \alpha}\left(\Phi^{n}\right) e^{-\lambda_{n}(T-t)} d t=-a_{n} e^{-\lambda_{n} T}=-\left\langle y^{0}, \Phi^{n} e^{-\lambda_{n} T}\right\rangle_{\mathcal{H}^{-s}, \mathcal{H}^{s}}
$$

Coming back to the definition given in (25) and taking into account that the solution of $-\partial_{t} \varphi-$ $\mathcal{A} \phi=0$ with final condition $\varphi^{T}=\Phi^{n}$ is exactly $\Phi^{n} e^{-\lambda_{n}(T-t)}$, we deduce that necessarily, the corresponding solution $y$ of (1) verifies

$$
\left\langle y(\cdot, T), \Phi^{n}\right\rangle_{\mathcal{H}^{-s}, \mathcal{H}^{s}}=0 \text { for every } n \in \mathbb{N} \backslash\{0\},
$$

which exactly means that $y(\cdot, T)=0$. Let us now estimate the control $u$. According to (53), by using (47), the fact that $f_{n}$ is the Fourier transform of $J_{n}$, and (50), we deduce that $u \in C^{0}([0, T])$ and

$$
\|u\|_{\infty} \leqslant F(\varepsilon, T, \alpha) \sum_{n \in \mathbb{N} \backslash\{0\}} \frac{a_{n}}{\left|\mathcal{O}_{\varepsilon, M, \alpha}\left(\Phi^{n}\right)\right|} \frac{1}{\lambda_{n}\left|\Lambda^{\prime}\left(i \lambda_{n}\right)\right|} \exp \left(3 \theta / 4-\lambda_{n} T / 2-\beta \lambda_{n} /(2 \sqrt{\theta+1})\right) .
$$

Let us estimate $\left|\mathcal{O}_{\varepsilon, M, \alpha}\left(\Phi^{n}\right) \lambda_{n} \Lambda^{\prime}\left(i \lambda_{n}\right)\right|$. Using the definition of $\Lambda$ given in (35), we observe that

$$
\Lambda^{\prime}\left(i \lambda_{n}\right)=\Gamma(\nu+1)\left(\frac{2}{j_{\nu, n}}\right)^{\nu} \frac{1}{2 \varepsilon \kappa^{2} j_{\nu, n}} J_{\nu}^{\prime}\left(j_{\nu, n}\right), \quad n \in \mathbb{N} \backslash\{0\} .
$$

Therefore, using (24) and (22), we deduce that

$$
\left|\mathcal{O}_{\varepsilon, L, M, \alpha}^{n} \lambda_{n} \Lambda^{\prime}\left(i \lambda_{n}\right)\right|=(1 / \varepsilon-\alpha)(2 \kappa)^{\frac{1}{2}} j_{\nu, n} .
$$

Hence, combining (55) and (54), we deduce that for $\varepsilon$ small enough

$$
\|u\|_{\infty} \leqslant F(\varepsilon, T, \alpha) e^{\frac{3 \theta}{4}} \sum_{n \in \mathbb{N} \backslash\{0\}} a_{n} e^{-\lambda_{n} T / 2} j_{\nu, n} \leqslant F(\varepsilon, T, \alpha) \exp \left(\frac{3 \theta}{4}-\lambda_{1} T / 2\right) .
$$


Using Cauchy-Schwarz inequality and the fact that $j_{\nu, n} \geqslant n \pi / 2$, we infer

$$
\|u\|_{\infty} \leqslant F(\varepsilon, T, \alpha)\left(\sum_{n \in \mathbb{N} \backslash\{0\}} a_{n}^{2}\right)^{\frac{1}{2}} \exp \left(\frac{3 \theta}{4}-\lambda_{1} T / 2\right),
$$

i.e.

$$
\|u\|_{\infty} \leqslant F(\varepsilon, T, \alpha) \exp \left(\frac{3 \theta}{4}-\lambda_{1} T / 2\right)\left\|y^{0}\right\|_{L^{2}\left((0,1) ; x^{-1 / \varepsilon} \mathrm{d} x\right)} .
$$

Using the expression of $\theta$ given in (45) and the definition of $\lambda_{1}$ given in (22), we deduce that (5) holds as soon as

$$
\frac{3}{4 \varepsilon \kappa^{2} T}-\frac{\varepsilon T \kappa^{2} j_{\nu, 1}^{2}}{2}<0
$$

Using the inequalities on $j_{\nu, 1}$ given in Lemma A.2 and taking into account the definition of $\nu$ and $\kappa$ given in (20), we obtain that (7) holds as soon as

$$
T>\frac{2 \sqrt{6}}{1-\alpha}>\frac{4.9}{1-\alpha} .
$$

Remark 3.1. According to the expression of u given in (53), u is of class $C^{\infty}$ and for every $k \in \mathbb{N}$, estimate (56) still holds if we consider the $W^{k, \infty}$-norm instead of the $L^{\infty}$-norm.

\subsection{Explosion of the cost of null controllability in small time}

During all the proof, we will consider $L=1$ and $M=1$ (see Remark 2.3). For the sake of simplicity we will simply denote by $K$ the cost of the control $\mathcal{K}(\varepsilon, T, 1,1, \alpha)$ and by $F(\varepsilon, T, \alpha)$ some fractional function of $(\varepsilon, T, \alpha)$ that may vary from line to line. Let $(\varepsilon, T, \alpha) \in(0,+\infty)^{2} \times(0,1)$ such that $1 / \varepsilon>\alpha$. Recall that $\nu$ and $\kappa$ are defined in (20). We define $y^{0} \in L^{2}\left((0,1) ; x^{-1 / \varepsilon} \mathrm{d} x\right)$ as follow:

$$
y^{0}(x):=x^{\frac{1}{2}(1 / \varepsilon-\alpha)} J_{\nu}\left(j_{\nu, 1} x^{\kappa}\right), \quad x \in(0,1) .
$$

From Lemma (A.3) we infer

$$
\int_{0}^{1}\left|y^{0}(x)\right|^{2} x^{-1 / \varepsilon} \mathrm{d} x=\int_{0}^{1} x^{-\alpha} J_{\nu}^{2}\left(j_{\nu, 1} x^{\kappa}\right) \mathrm{d} x=\frac{\left[J_{\nu}^{\prime}\left(j_{\nu, 1}\right)\right]^{2}}{2 \kappa} .
$$

Let us consider some small parameter $\eta>0$. We consider $u \in U\left(\varepsilon, T, \alpha, y^{0}\right)$ such that (remind that the quantity $K$ is not necessarily reached for some control $u$ here)

$$
y(\cdot, T) \equiv 0 \quad \text { and } \quad\|u\|_{L^{2}(0, T)} \leq(\mathcal{K}+\eta)\left\|y_{0}\right\|_{L^{2}\left((0,1) ; x^{-1 / \varepsilon} \mathrm{d} x\right)} .
$$

For any $n \in \mathbb{N} \backslash\{0\}$, let us define $z^{n}:[0, L] \times[0, T] \mapsto \mathbb{R}$ by

$$
z^{n}(x, t):=x^{\frac{1}{2}(1 / \varepsilon-\alpha)} J_{\nu}\left(j_{\nu, n} x^{\kappa}\right) \exp \left(\varepsilon\left(\kappa j_{\nu, n}\right)^{2}(t-T)\right), \quad(x, t) \in(0,1) \times(0, T) .
$$

Then, it is not difficult to check that $z^{n}(t) \in \mathcal{H}^{s}$ for any $s \geq 0$ and

$$
\begin{gathered}
-z_{t}^{n}-\varepsilon\left(x^{\alpha+1} z_{x}^{n}\right)_{x}+x^{\alpha} z_{x}^{n}=0, \quad(x, t) \in(0,1) \times(0, T), \\
z^{n}(0, t)=0, \quad z^{n}(1, t)=0, \quad t \in(0, T) .
\end{gathered}
$$

Moreover, from (25) we get, for some $s \geq \nu-1 / 2$,

$$
\left\langle y^{0}, z^{n}(0)\right\rangle_{\mathcal{H}^{-s}, \mathcal{H}^{s}}=-\int_{0}^{T} u(t) \mathcal{O}_{\varepsilon, 1, \alpha}\left(z^{n}\right)(t) \mathrm{d} t .
$$

Then, using (59), (64), Lemma (A.3) and Lemma 2.1, we get that for all $n \in \mathbb{N} \backslash\{0\}$

$$
\frac{(1 / \varepsilon-\alpha)\left(j_{\nu, n}\right)^{\nu}}{2^{\nu} \Gamma(\nu+1)} \int_{0}^{T} u(t) \exp \left(\varepsilon\left(\kappa j_{\nu, n}\right)^{2} t\right) \mathrm{d} t=-\delta_{1 n} \frac{\left[J_{\nu}^{\prime}\left(j_{\nu, 1}\right)\right]^{2}}{2 \kappa}
$$


Next we introduce the function $v: \mathbb{C} \rightarrow \mathbb{C}$ defined by

$$
v(s):=\int_{-T / 2}^{-T / 2} u\left(t+\frac{T}{2}\right) e^{-i s t} \mathrm{~d} t, \quad s \in \mathbb{C} .
$$

Then, it is not difficult to see from (65) and (66) that we have

$$
v\left(i \varepsilon\left(\kappa j_{\nu, n}\right)^{2}\right)=0 \quad \text { if } \quad n \in \mathbb{N} \backslash\{0,1\}
$$

and

$$
v\left(i \varepsilon\left(\kappa j_{\nu, 1}\right)^{2}\right)=-\frac{2^{\nu} \Gamma(\nu+1)\left[J_{\nu}^{\prime}\left(j_{\nu, 1}\right)\right]^{2}}{(1-\alpha)(1 / \varepsilon-\alpha)\left(j_{\nu, 1}\right)^{\nu}} \exp \left(-\frac{\varepsilon\left(\kappa j_{\nu, 1}\right)^{2} T}{2}\right) .
$$

On the other hand, we readily have

$$
|v(s)| \leq \exp \left(\frac{T|\Im(s)|}{2}\right) \int_{0}^{T}|u(t)| \mathrm{d} t \leq(\mathcal{K}+\eta) T^{1 / 2} \exp \left(\frac{T|\Im(s)|}{2}\right)\left\|y^{0}\right\|_{L^{2}\left((0,1) ; x^{-1 / \varepsilon}\right)} \mathrm{d} x .
$$

Let us introduce the function $f: \mathbb{C} \rightarrow \mathbb{C}$ defined by

$$
f(s):=v\left(\frac{s-i \delta}{\varepsilon}\right), \quad s \in \mathbb{C},
$$

for some $\delta>0$ that will be chosen later on. Then, it is not difficult to see that $f$ is an entire function satisfying

$$
f\left(a_{k}\right)=0, \quad k \in \mathbb{N} \backslash\{0,1\}, \quad \text { with } \quad a_{k}:=i\left(\left(\varepsilon \kappa j_{\nu, k}\right)^{2}+\delta\right), \quad k \in \mathbb{N} \backslash\{0\} .
$$

Moreover, thanks to (68) and (69) we infer

$$
f\left(a_{1}\right)=-\frac{2^{\nu} \Gamma(\nu+1)\left[J_{\nu}^{\prime}\left(j_{\nu, 1}\right)\right]^{2}}{(1-\alpha)(1 / \varepsilon-\alpha)\left(j_{\nu, 1}\right)^{\nu}} \exp \left(-\frac{\varepsilon\left(\kappa j_{\nu, 1}\right)^{2} T}{2}\right)
$$

and

$$
\log |f(s)| \leq \frac{T|\Im(s)-\delta|}{2 \varepsilon}+\log \left((\mathcal{K}+\eta) T^{1 / 2} \frac{\left|J_{\nu}^{\prime}\left(j_{\nu, 1}\right)\right|}{\sqrt{2 \kappa}}\right) .
$$

Before going further let us recall the following representation theorem [28, p. 56].

Theorem 3.1. Let $g(z)$ be an entire function of exponential type and suppose that

$$
\int_{-\infty}^{+\infty} \frac{\log ^{+}|g(x)|}{1+x^{2}} d x<\infty
$$

Denote by $\left\{b_{\ell}\right\}_{\ell \in \mathbb{N}}$ the set of zeros of $g(z)$ lying in $\Im(z)>0$ (each zero being repeated according to its multiplicity). Then, for $\Im(z)>0$

$$
\log |g(z)|=\mathcal{A} \Im(z)+\sum_{\ell=1}^{\infty} \log \left|\frac{z-b_{\ell}}{z-\bar{b}_{\ell}}\right|+\frac{\Im(z)}{\pi} \int_{-\infty}^{+\infty} \frac{\log |g(s)|}{|s-z|^{2}} d s
$$

where

$$
\mathcal{A}=\limsup _{y \rightarrow+\infty} \frac{\log |g(i y)|}{y} .
$$

We apply Theorem 3.1 to the function $f$ defined in (70) and we obtain

$$
\log \left|f\left(a_{1}\right)\right|=\frac{\left(\left(\varepsilon \kappa j_{\nu, 1}\right)^{2}+\delta\right) T}{2 \varepsilon}+\sum_{\ell=2}^{\infty} \log \left|\frac{a_{1}-a_{\ell}}{a_{1}-\bar{a}_{\ell}}\right|+\frac{\Im\left(a_{1}\right)}{\pi} \int_{-\infty}^{+\infty} \frac{\log |f(s)|}{\left|s-a_{1}\right|^{2}} \mathrm{~d} s .
$$

Thanks to (71), and the fact that $\Im\left(a_{\ell}\right)>0$ for $\ell \in \mathbb{N} \backslash\{0,1\}$ we have 


$$
\begin{aligned}
\sum_{\ell=2}^{\infty} \log \left|\frac{a_{1}-a_{\ell}}{a_{1}-\bar{a}_{\ell}}\right|= & \sum_{\ell=2}^{\infty} \log \left(\frac{\left(\varepsilon j_{\nu, \ell}\right)^{2}-\left(\varepsilon j_{\nu, 1}\right)^{2}}{2 \delta / \kappa^{2}+\left(\varepsilon j_{\nu, 1}\right)^{2}+\left(\varepsilon j_{\nu, \ell}\right)^{2}}\right) \\
& \leq \sum_{\ell=2}^{\infty} \frac{1}{j_{\nu, \ell+1}-j_{\nu, l}} \int_{j_{\nu, l}}^{j_{\nu, l+1}} \log \left(\frac{\varepsilon^{2} x^{2}}{2 \delta / \kappa^{2}+\varepsilon^{2} x^{2}}\right) \mathrm{d} x \\
& \leq \frac{1}{\pi} \int_{j_{\nu, 2}}^{\infty} \log \left(\frac{\varepsilon^{2} x^{2}}{2 \delta / \kappa^{2}+\varepsilon^{2} x^{2}}\right) \mathrm{d} x
\end{aligned}
$$

where we have used Lemma A.2. Now, we make the change of variables

$$
\tau=\frac{\varepsilon \kappa}{\sqrt{2 \delta}} x
$$

then performing integration by parts we obtain

$$
\begin{gathered}
\frac{\sqrt{2 \delta}}{\varepsilon \kappa} \int_{\varepsilon \kappa j_{\nu, 2} / \sqrt{2 \delta}}^{\infty} \log \left(\frac{\tau^{2}}{1+\tau^{2}}\right) \mathrm{d} \tau \\
=-j_{\nu, 2} \log \left(\frac{1}{1+2 \delta /\left(\varepsilon \kappa j_{\nu, 2}\right)^{2}}\right)-2 \frac{\sqrt{2 \delta}}{\varepsilon \kappa} \int_{\varepsilon \kappa j_{\nu, 2} / \sqrt{2 \delta}}^{\infty} \frac{1}{1+\tau^{2}} \mathrm{~d} \tau \\
=-j_{\nu, 2} \log \left(\frac{1}{1+2 \delta /\left(\varepsilon \kappa j_{\nu, 2}\right)^{2}}\right)-2 \frac{\sqrt{2 \delta}}{\varepsilon \kappa}\left(\frac{\pi}{2}-\tan ^{-1}\left(\varepsilon \kappa j_{\nu, 2} / \sqrt{2 \delta}\right)\right) .
\end{gathered}
$$

Thus, we get for any $(\varepsilon, T, \alpha) \in(0,+\infty)^{2} \times(0,1)$ such that $1 / \varepsilon>\alpha$ that

$$
\sum_{\ell=2}^{\infty} \log \left|\frac{a_{1}-a_{\ell}}{a_{1}-\bar{a}_{\ell}}\right| \leq-\frac{\sqrt{2 \delta}}{\varepsilon \kappa}+\frac{j_{\nu, 2}}{\pi} \log \left(1+2 \delta /\left(\varepsilon \kappa j_{\nu, 2}\right)^{2}\right)+\frac{2 \sqrt{2 \delta}}{\varepsilon \kappa \pi} \tan ^{-1}\left(\varepsilon \kappa j_{\nu, 2} / \sqrt{2 \delta}\right) .
$$

On the other hand, straightforward computations using (73) give for some $C>0$

$$
\frac{\Im\left(a_{1}\right)}{\pi} \int_{-\infty}^{+\infty} \frac{\log |f(s)|}{\left|s-a_{1}\right|^{2}} \mathrm{~d} s \leq \frac{T \delta}{2 \varepsilon}+\log \left((\mathcal{K}+\eta) T^{1 / 2} \frac{\left|J_{\nu}^{\prime}\left(j_{\nu, 1}\right)\right|}{\sqrt{2 \kappa}}\right)+C .
$$

At this point, we have for any $\delta>0$ and some $C>0$ independent of $(\varepsilon, T, \alpha)$

$$
\begin{gathered}
\log \left(\frac{2^{\nu} \Gamma(\nu+1)\left[J_{\nu}^{\prime}\left(j_{\nu, 1}\right)\right]^{2}}{(1-\alpha)(1 / \varepsilon-\alpha)\left(j_{\nu, 1}\right)^{\nu}}\right)-\frac{\varepsilon\left(\kappa j_{\nu, 1}\right)^{2} T}{2} \leq \frac{\left(\left(\varepsilon \kappa j_{\nu, 1}\right)^{2}+\delta\right) T}{2 \varepsilon}+\frac{2 \sqrt{2 \delta}}{\varepsilon \kappa \pi} \tan ^{-1}\left(\varepsilon \kappa j_{\nu, 2} / \sqrt{2 \delta}\right) \\
-\frac{\sqrt{2 \delta}}{\varepsilon \kappa}+\frac{j_{\nu, 2}}{\pi} \log \left(1+2 \delta /\left(\varepsilon \kappa j_{\nu, 2}\right)^{2}\right)+\frac{T \delta}{2 \varepsilon}+\log \left((K+\eta) T^{1 / 2} \frac{\left|J_{\nu}^{\prime}\left(j_{\nu, 1}\right)\right|}{\sqrt{2 \kappa}}\right)+C .
\end{gathered}
$$

Taking the exponential in (80), we obtain

$$
\begin{array}{r}
\exp \left(-\left(\varepsilon\left(\kappa j_{\nu, 1}\right)^{2}+\frac{\delta}{\varepsilon}\right) T+\frac{\sqrt{2 \delta}}{\varepsilon \kappa}-\frac{j_{\nu, 2}}{\pi} \log \left(1+2 \delta /\left(\varepsilon \kappa j_{\nu, 2}\right)^{2}\right)-\frac{2 \sqrt{2 \delta}}{\varepsilon \kappa \pi} \tan ^{-1}\left(\frac{\varepsilon \kappa j_{\nu, 2}}{\sqrt{2 \delta}}\right)\right) \\
\leq F(\varepsilon, T, \alpha)(\mathcal{K}+\eta) \frac{\left|J_{\nu}^{\prime}\left(j_{\nu, 1}\right)\right|\left(j_{\nu, 1}\right)^{\nu}}{2^{\nu} \Gamma(\nu+1)}
\end{array}
$$

Using Lemma A.2 and making use of the definition of $\nu$ in (20), we obtain

$$
j_{\nu, n} \sim \frac{1}{\varepsilon(1-\alpha)} \quad \text { as } \quad \varepsilon \rightarrow 0^{+}, \quad n=1,2 .
$$

Hence, for $\varepsilon$ small enough one gets from (81) that (remind the definition of $\kappa$ given in (20))

$$
\begin{array}{r}
\exp \left(\frac{1}{\varepsilon}\left(-\frac{T}{4}-\delta T+\frac{2 \sqrt{2 \delta}}{1-\alpha}-\frac{\ln (1+8 \delta)}{(1-\alpha) \pi}-\frac{4 \sqrt{2 \delta}}{(1-\alpha) \pi} \tan ^{-1}\left(\frac{1}{2 \sqrt{2 \delta}}\right)\right)\right) \\
\leq F(\varepsilon, T, \alpha)(\mathcal{K}+\eta) \frac{\left|J_{\nu}^{\prime}\left(j_{\nu, 1}\right)\right|\left(j_{\nu, 1}\right)^{\nu}}{2^{\nu} \Gamma(\nu+1)}
\end{array}
$$


However, there is a hidden leading coefficient in the right hand side of (82). Indeed, Using Lemma A.4 and the Stirling formula (see for example [1, 6.1.39]), we see that

$$
\frac{2^{\nu} \Gamma(\nu+1)}{\left|J_{\nu}^{\prime}\left(j_{\nu, 1}\right)\right|\left(j_{\nu, 1}\right)^{\nu}} \sim F(\varepsilon, T, \alpha)\left(\frac{2}{e}\right)^{\nu} \quad \text { as } \quad \varepsilon \rightarrow 0^{+} .
$$

We deduce, using (83) together with (82), that

$$
\begin{array}{r}
\exp \left(\frac{1}{\varepsilon}\left(\frac{\ln (2)-1}{1-\alpha}-\frac{T}{4}-\delta T+\frac{2 \sqrt{2 \delta}}{1-\alpha}-\frac{\ln (1+8 \delta)}{(1-\alpha) \pi}-\frac{4 \sqrt{2 \delta}}{(1-\alpha) \pi} \tan ^{-1}\left(\frac{1}{2 \sqrt{2 \delta}}\right)\right)\right) \\
\leq F(\varepsilon, T, \alpha)(K+\eta)
\end{array}
$$

Finally, by taking $\eta$ small enough, we deduce(7) holds as soon as

$$
\frac{\ln (2)-1}{(1-\alpha)}-\frac{T}{4}-\delta T+\frac{2 \sqrt{2 \delta}}{(1-\alpha)}-\frac{\ln (1+8 \delta)}{(1-\alpha) \pi}-\frac{4 \sqrt{2 \delta}}{(1-\alpha) \pi} \tan ^{-1}\left(\frac{1}{2 \sqrt{2 \delta}}\right)>0
$$

i.e.

$$
T<\frac{4\left(-\pi+2 \pi \sqrt{2 \delta}-4 \sqrt{2 \delta} \tan ^{-1}\left(\frac{1}{2 \sqrt{2 \delta}}\right)+\pi \ln (2)-\ln (1+8 \delta)\right)}{(1-\alpha) \pi(1+4 \delta)} .
$$

Maximizing the right-hand side with respect to $\delta$ and taking a numerical approximation of the maximum (reached for $\delta \simeq 1.37$ ), we infer the result.

\section{Appendix}

\section{A Bessel functions}

Bessel functions of order $\nu$ are solutions of the differential equation

$$
x^{2} \frac{d^{2} y}{d x^{2}}+x \frac{d y}{d x}+\left(x^{2}-\nu^{2}\right) y=0, \quad x \in(0, \infty) .
$$

The Bessel functions $J_{\nu}$ of the first kind that are finite at the origin, the Bessel functions $Y_{\nu}$ of the second kind, that are linearly independent of $J_{\nu}$. Only the first type of Bessel functions will be relevant to our analysis.

It is possible to define the Bessel functions of the first kind by their Taylor series expansion around $x=0$ (see [33] for various way to define the Bessel functions)

$$
J_{\nu}(x)=\sum_{m \geq 0} \frac{(-1)^{m}}{m ! \Gamma(m+\nu+1)}\left(\frac{x}{2}\right)^{2 m+\nu}, \quad x \geq 0
$$

where $\Gamma(\cdot)$ is the Gamma function. In particular, for $\nu \in \mathbb{R} \backslash\left\{-\mathbb{N}^{*}\right\}$ and $0<x \leq \sqrt{\nu+1}$, from (85) one can obtain (see $[1,9.1 .7$, p. 360]):

$$
J_{\nu}(x) \sim \frac{1}{\Gamma(\nu+1)}\left(\frac{x}{2}\right)^{\nu} \quad \text { as } \quad x \rightarrow 0^{+} .
$$

Besides, Bessel functions of the first kind satisfy the recurrence formula [1, 9.1.27]:

$$
x J_{\nu}^{\prime}(x)-\nu J_{\nu}(x)=-x J_{\nu+1}(x) .
$$

Let us now recall the following well-known asymptotic behavior of Bessel function $J_{\nu}$

Lemma A.1. [19, Lemma 7.2, p.129] For any $\nu \in \mathbb{R}$

$$
J_{\nu}(x)=\sqrt{\frac{2}{\pi x}}\left\{\cos \left(x-\frac{\nu \pi}{2}-\frac{\pi}{4}\right)+\mathcal{O}\left(\frac{1}{x}\right)\right\} \quad \text { as } \quad x \rightarrow \infty .
$$

The function $J_{\nu}$, for $\nu \geq-1$, has an infinite number of real zeros $j_{\nu, 1}<j_{\nu, 2}<\cdots$, all of which are simple, with the possible exception of $x=0$. We have the following informations on the location of the zeros of the Bessel functions $J_{\nu}$ : 
Lemma A.2. [19, Proposition 7.8] Let $\nu \geq 0$.

1. The difference sequence $\left(j_{\nu, n+1}-j_{\nu, n}\right)_{n}$ converges to $\pi$ as $n \rightarrow+\infty$.

2. The sequence $\left(j_{\nu, n+1}-j_{\nu, n}\right)_{n}$ is strictly decreasing if $|\nu|>\frac{1}{2}$, strictly increasing if $|\nu|<\frac{1}{2}$, and constant if $|\nu|=\frac{1}{2}$.

3. For $\nu$ large enough, one has

$$
\nu+\nu^{\frac{1}{3}}<j_{\nu, 1}<\nu+4 \nu^{\frac{1}{3}}
$$

The Bessel functions enjoy the following orthogonality property in $[0,1]$.

Lemma A.3. For any $\nu \geq-1 / 2$, (see [27, ]):

$$
\int_{0}^{1} x J_{\nu}\left(j_{\nu, n} x\right) J_{\nu}\left(j_{\nu, m} x\right) d x=\frac{\delta_{n m}}{2}\left[J_{\nu}^{\prime}\left(j_{\nu, n}\right)\right]^{2} .
$$

(89) allow to prove that the family

$$
\left\{\frac{\sqrt{2 y}}{\left|J_{\nu}^{\prime}\left(j_{\nu, n}\right)\right|} J_{\nu}\left(j_{\nu, n} y\right)\right\}_{n \in \mathbb{N} \backslash\{0\}}
$$

forms an orthonormal basis of $L^{2}(0,1)$.

Lemma A.4. For any $\nu \geq 0$ and any $n \in \mathbb{N} \backslash\{0\}$ we have

$$
\sqrt{j_{\nu, n}}\left|J_{\nu}^{\prime}\left(j_{\nu, n}\right)\right|=\sqrt{\frac{2}{\pi}}+O_{n \rightarrow \infty}\left(\frac{1}{j_{\nu, n}}\right) .
$$

The proof of this result is left to the reader using Lemma A.1 and the recurrence formula(87).

\section{B The Transport equation}

Let $T>0, L>0$ and $\alpha \in(0,1)$. We aim at giving a meaning to the following transport equation:

$$
\begin{cases}y_{t}+M x^{\alpha} y_{x}=0 & \text { in }(0, L) \times(0, T), \\ y(0, t)=u(t) & \text { on }(0, T), \\ y(x, 0)=y^{0}(x) & \text { in }(0, L) .\end{cases}
$$

In all what follows, a function defined on some subset of $\mathbb{R}$ will always be extended on $\mathbb{R}$ by 0 .

We define the following spaces of regular functions :

$$
C_{\alpha}([0, T] \times[0, L]):=\left\{g \in C^{0}([0, T] \times[0, L]) \mid g_{t} \in C^{0}([0, T] \times[0, L]), x^{\alpha} g_{x} \in C^{0}([0, T] \times[0, L])\right\}
$$

and

$$
S_{\alpha}((0, T) \times(0, L)):=W^{1,1}\left((0, T), L^{1}(0, L)\right) \cap L^{1}\left((0, T), W_{\alpha}^{1}(0, L)\right),
$$

where

$$
W_{\alpha}^{1}(0, L):=\left\{\varphi \in L^{1}(0, L) \mid\left(x^{\alpha} \varphi\right)_{x} \in L^{1}(0, L)\right\} .
$$

One observes that functions $g \in S_{\alpha}((0, T) \times(0, L))$ are notably such that

$$
x^{\alpha} g \in C^{0}\left([0, L], L^{1}(0, T)\right), g \in C^{0}\left([0, T], L^{1}(0, L)\right) .
$$

We will assume from now on that

$$
y^{0} \in L^{\infty}(0, L), u \in L^{\infty}(0, T) .
$$


If $y \in C_{\alpha}([0, T] \times[0, L])$ and verifies (91) everywhere, then necessarily $y^{0} \in C^{0}([0, L])$, $u \in C^{0}([0, T])$ and $y^{0}(0)=u(0)$. Then, for every $\varphi \in S_{\alpha}((0, T) \times(0, L))$, one has, using integrations by parts and (95),

$$
\begin{aligned}
-\int_{0}^{T} \int_{0}^{L} y\left(\varphi_{t}+\left(x^{\alpha} \varphi\right)_{x}\right) d x d t+ & \int_{0}^{T} y(L, t) L^{\alpha} \varphi(L, t) d t-\int_{0}^{T} u(t)\left\{x^{\alpha} \varphi(x, t)\right\}_{\mid x=0} d t \\
& +\int_{0}^{L} y(x, T) \varphi(x, T) d x-\int_{0}^{L} y^{0}(x) \varphi(x, 0) d x=0 .
\end{aligned}
$$

This computation justifies the following definition:

Definition B.1. A weak solution of the Cauchy problem (91) is a function $y \in L^{\infty}((0, T) \times$ $(0, L))$ such that, for every $\varphi \in S_{\alpha}((0, T) \times(0, L))$ verifying moreover

$$
\begin{aligned}
& \varphi(L, \cdot)=0 \text { in }(0, T), \\
& \varphi(\cdot, T)=0 \text { in }(0, L),
\end{aligned}
$$

one has

$$
-\int_{0}^{T} \int_{0}^{L}\left(\varphi_{t}+\left(x^{\alpha} \varphi\right)_{x}\right) y d x d t-\int_{0}^{T} u(t)\left\{x^{\alpha} \varphi(x, t)\right\}_{\mid x=0} d t-\int_{0}^{L} y^{0}(x) \varphi(x, 0) d x=0 .
$$

We are now going to prove the following well-posedness result for the Cauchy problem (91):

Theorem B.1. The Cauchy Problem (91) has a unique weak solution verifying moreover

$$
\|y\|_{L^{\infty}((0, T) \times(0, L))} \leqslant \max \left(\left\|y^{0}\right\|_{L^{\infty}(0, L)},\|u\|_{L^{\infty}(0, L)}\right)
$$

Proof of Theorem B.1. One can compute explicitly the characteristics curves of (91): let $x_{0} \in(0, L)$, the solutions of the ordinary differential equation

$$
\left\{\begin{aligned}
x^{\prime} & =x^{\alpha} \\
x(0) & =x^{0}
\end{aligned}\right.
$$

are given in the following way for every $t \in \mathbb{R}$ :

$$
x(t)=\left(x_{0}^{1-\alpha}+(1-\alpha) t\right)^{\frac{1}{1-\alpha}}, t>-\frac{x_{0}^{1-\alpha}}{1-\alpha}, x(t)=0, t \leqslant-\frac{x_{0}^{1-\alpha}}{1-\alpha} .
$$

Remark B.1. We remark that the characteristic curves intersect (and are even mixed up), but only for $x=0$. There is some hope to use these characteristic curves to exhibit some solutions because for every point in $(0, T) \times(0, L)$, there exists a unique characteristic curve coming to that point.

Existence. we consider

$$
y(t, x):=y^{0}\left(\left(x^{1-\alpha}-t(1-\alpha)\right)^{\frac{1}{1-\alpha}}\right)+u\left(\left(t-\frac{x^{1-\alpha}}{1-\alpha}\right) .\right.
$$

One readily verifies that $y \in L^{\infty}((0, T) \times(0, L))$. Let us know introduce $\left(y_{n}^{0}\right)_{n \in \mathbb{N}}$ a sequence of functions in $C_{0}^{1}([0, L])$ converging to $y^{0}$ in $L^{\infty}(0, L)$ for the weak-* topology and $\left(u_{n}\right)_{n \in \mathbb{N}}$ a sequence of functions in $C_{0}^{1}(0, T)$ converging to $u$ in $L^{\infty}(0, T)$ for the weak-* topology. Let

$$
y_{n}(t, x):=y_{n}^{0}\left(\left(x^{1-\alpha}-t(1-\alpha)\right)^{\frac{1}{1-\alpha}}\right)+u_{n}\left(t-\frac{x^{1-\alpha}}{1-\alpha}\right) .
$$

$y_{n}$ is differentiable with respect to $t, x^{\alpha} \partial_{x} y_{n}$ has a meaning at every point $(t, x)$, and one has

$$
\begin{aligned}
\partial_{t} y_{n}(t, x) & =-\left(x^{1-\alpha}+(1-\alpha) t\right)^{\frac{\alpha}{1-\alpha}} y_{n}^{0^{\prime}}\left(\left(x^{1-\alpha}-t(1-\alpha)\right)^{\frac{1}{1-\alpha}}\right)+u_{n}^{\prime}\left(t-\frac{x^{1-\alpha}}{1-\alpha}\right) \\
& =-x^{\alpha} \partial_{x} y_{n}(t, x) .
\end{aligned}
$$


This implies that $y_{n}$ satisfies $(91)$ in the classical sense and one verifies that $y \in C_{\alpha}([0, T] \times[0, L])$. From equality (102), we obtain

$$
\left\|y_{n}\right\|_{L^{\infty}((0, T) \times(0, L))} \leqslant \max \left(\left\|y_{n}^{0}\right\|_{L^{\infty}(0, L)},\left\|u_{n}\right\|_{L^{\infty}(0, T)}\right) .
$$

Since the sequences $\left(y_{n}^{0}\right)_{n \in \mathbb{N}}$ and $\left(u_{n}\right)_{n \in \mathbb{N}}$ converge for weak- ${ }^{*} L^{\infty}$-topology, theses sequences are bounded for the strong $L^{\infty}$-topology. This implies that $\left(y_{n}\right)$ is bounded in $L^{\infty}((0, T) \times(0, L))$, hence, up to some subsequence $y_{n}{ }^{*} y$ in the weak-* $L^{\infty}$-topology.

The only thing that remains to prove is that $y$ is a weak solution of (91). Using that for all $n, y_{n}$ is a weak solution of $(91)$, we obtain that for every $\varphi \in S_{\alpha}((0, T) \times(0, L))$ verifying moreover (96) and (97),

$$
-\int_{0}^{T} \int_{0}^{L}\left(\varphi_{t}+\left(x^{\alpha} \varphi\right)_{x}\right) y_{n} d x d t-\int_{0}^{T} u_{n}(t)\left(x^{\alpha} \varphi(t, x)\right)_{\mid x=0} d t-\int_{0}^{L} y_{n}^{0}(x) \varphi(0, x)=0 .
$$

One can make $n \rightarrow \infty$ in this equality (see notably (94)). We obtain that $y$ is a weak solution of (91).

Uniqueness. Let $y_{1}$ and $y_{2}$ be two solutions of (91) (with initial condition $y^{0}$ and boundary condition $u$ ) and let us consider $y:=y_{1}-y_{2} . y$ is a weak solution of

$$
\left\{\begin{array}{r}
y_{t}+x^{\alpha} y_{x}=0 \text { in }(0, T) \times(0, L), \\
y(0, x)=0 \text { in }(0, L), \\
y(t, 0)=0 \text { in }(0, T) .
\end{array}\right.
$$

We want to prove that necessarily $y \equiv 0$. Using (103) and (98), necessarily for every $\varphi \in$ $S_{\alpha}((0, T) \times(0, L))$ verifying $(96)$ and $(97)$,

$$
-\int_{0}^{T} \int_{0}^{L}\left(\varphi_{t}+\left(x^{\alpha} \varphi\right)_{x}\right) y \mathrm{~d} x \mathrm{~d} t=0
$$

Let us consider some $\Phi \in C_{0}^{\infty}((0, T) \times(0, L))$. We want to solve the following backward problem:

$$
\left\{\begin{aligned}
z_{t}+\left(x^{\alpha} z\right)_{x}=\Phi & \text { in }(0, T) \times(0, L), \\
z(T, x)=0 & \text { in }(0, L), \\
z(t, L)=0 & \text { in }(0, T) .
\end{aligned}\right.
$$

We define a solution of (105) in the following way:

$$
\begin{aligned}
& z(t, x)=-\int_{0}^{T-t} \frac{\left(x^{1-\alpha}+(1-\alpha)(T-t-r)\right)^{\frac{\alpha}{1-\alpha}}}{x^{\alpha}} \Phi\left(T-r,\left(x^{1-\alpha}\right.\right. \\
& \left.+(1-\alpha)(T-t-r))^{\frac{1}{1-\alpha}}\right) \mathrm{d} r .
\end{aligned}
$$

One readily verifies that $z$ verifies $(96)$ and (97). What is not clear is whether $z \in S_{\alpha}((0, T) \times$ $(0, L))$. One has for every $t$ and $x$

$$
|z(t, x)| \leqslant \frac{C(T, L)\|\Phi\|_{\infty}}{x^{\alpha}}
$$

which implies that $z \in L^{1}((0, T) \times(0, L))$. Since $\Phi \in C_{0}^{\infty}((0, T) \times(0, L)$ and $z$ verifies $(105)$ in $\mathcal{D}^{\prime}\left((0, T) \times(0, L)\right.$, as soon as $\left(x^{\alpha} z\right)_{x} \in L^{1}\left((0, T) \times(0, L)\right.$, we have $z_{t} \in L^{1}((0, T) \times(0, L)$. Let us compute

$$
\begin{aligned}
& \left.\left(x^{\alpha} z\right)_{x}(t, x)=-\int_{0}^{T-t} \alpha x^{-\alpha}\left(x^{1-\alpha}+(1-\alpha)(T-t-r)\right)^{\frac{2 \alpha-1}{1-\alpha}}\right) \Phi\left(T-r,\left(x^{1-\alpha}\right.\right. \\
& \left.\left.+(1-\alpha)(T-t-r))^{\frac{1}{1-\alpha}}\right) d r-\int_{0}^{T-t} x^{-\alpha}\left(x^{1-\alpha}+(1-\alpha)(T-t-r)\right)^{\frac{2 \alpha}{1-\alpha}}\right) \\
& \Phi_{x}\left(T-r,\left(x^{1-\alpha}+(1-\alpha)(T-t-r)\right)^{\frac{1}{1-\alpha}}\right) \mathrm{d} r .
\end{aligned}
$$


For $\alpha \geqslant 1 / 2$ we have

$$
\begin{aligned}
& \left.\mid-\int_{0}^{T-t} \alpha x^{-\alpha}\left(x^{1-\alpha}+(1-\alpha)(T-t-r)\right)^{\frac{2 \alpha-1}{1-\alpha}}\right) \Phi\left(T-r,\left(x^{1-\alpha}+(1-\alpha)(T-t\right.\right. \\
& \left.-r))^{\frac{1}{1-\alpha}}\right) d r \mid \leqslant C(T, L)\|\Phi\|_{\infty} x^{-\alpha}
\end{aligned}
$$

and for $\alpha \leqslant 1 / 2$ we have

$$
\begin{aligned}
& \left.\mid-\int_{0}^{T-t} \alpha x^{-\alpha}\left(x^{1-\alpha}+(1-\alpha)(T-t-r)\right)^{\frac{2 \alpha-1}{1-\alpha}}\right) \Phi\left(T-r,\left(x^{1-\alpha}+(1-\alpha)(T-t\right.\right. \\
& \left.-r))^{\frac{1}{1-\alpha}}\right) d r \mid \leqslant C(T, L)\|\Phi\|_{\infty} x^{\alpha-1} .
\end{aligned}
$$

We also have

$$
\begin{aligned}
& \left|-\int_{0}^{T-t} x^{-\alpha}\left(x^{1-\alpha}+(1-\alpha)(T-t-r)\right)^{\frac{2 \alpha}{1-\alpha}} \Phi_{x}\left(T-r,\left(x^{1-\alpha}+(1-\alpha)(T-t r)\right)^{\frac{1}{1-\alpha}}\right) d r\right| \\
& \leqslant C(T, L)\left\|\Phi_{x}\right\|_{\infty} x^{-\alpha}
\end{aligned}
$$

so that $\left(x^{\alpha} z\right)_{x} \in L^{1}(0, T)$. We deduce that in both cases $\left(x^{\alpha} z\right)_{x} \in L^{1}((0, T) \times(0, L))$. We deduce that $z \in S_{\alpha}((0, T) \times(0, L))$ as wanted, and the desired result follows.

\section{References}

[1] M. Abramowitz and I. A. Stegun, Handbook of mathematical functions with formulas, graphs and mathematical tables, National Bureau os Standards. App. Math. series, Vol. 55. 1964.

[2] L. Ambrosio, Transport equation and Cauchy Problem for BV vector fields, Inventiones Mathematicae 158 (2004), 227-260.

[3] S. A. Avdonin and S. A. Ivanov, Families of Exponentials: The Method of Moments in Controllability Problems for Distributed Parameter Systems. Cambridge New York, Cambridge University Press, 1995.

[4] P. Cannarsa, P. Martinez and J. Vancostenoble, Carleman estimates for a class of degenerate parabolic operators, SIAM J. Control Optim., 47, 1 (2008) 1-19 .

[5] P. Cannarsa, J. Tort and M. Yamamoto, Unique continuation and approximate controllability for a degenerate parabolic equation, Applicable analysis, (2011).

[6] N. Carreno and S. Guerrero, On the non-uniform null controllability of a linear KdV equation. Asymptot. Anal. 2015;94(1-2):33-69.

[7] J.-M. Coron and Sergio Guerrero, Singular optimal control: A linear 1-D parabolic-hyperbolic example, Asymptotic Analysis 44 (2005), 237-257.

[8] R. J. DiPerna and P.-L. Lions, Ordinary differential equations, transport theory and Sobolev spaces, Inventiones Mathematicae 98 (1989), no. 3, 511-547.

[9] H. O. Fattorini and D. L. Russell, Exact controllability theorems for linear parabolic equations in one space dimension, Archive for Rational Mechanics and Analysis. 43 (1971) 272-292.

[10] A. V. Fursikov and O. Y. Imanuvilov, Controllability of Evolution Equations, Lecture Notes \#34, Seoul National University, Korea, 1996.

[11] O. Glass, A complex-analytic approach to the problem of uniform controllability of a transport equation in the vanishing viscosity limit. J. Funct. Anal. 258 (2010), no. 3, 852-868.

[12] O. Glass and S. Guerrero, On the uniform controllability of the Burgers equation. SIAM J. Control Optim. 46 (2007), no. 4, 1211-1238.

[13] O. Glass and S. Guerrero, Uniform controllability of a transport equation in zero diffusion-dispersion limit. Math. Models Methods Appl. Sci. 19 (2009), no. 9, 1567-1601.

[14] S. Guerrero and G. Lebeau, Singular optimal control for a transport-diffusion equation. Comm. Partial Differential Equations 32 (2007), no. 10-12, 1813-1836.

[15] M. Gueye, Exact boundary controllability of $1-D$ parabolic and hyperbolic equations, SIAM J. Control Optim., Vol. 52, No. 4, (2014) 2037-2054.

[16] E. Kamke, Differentialgleichungen: Lösungsmethoden und lösungen, $3^{\text {rd }}$ edition, Chelsea Publishing Company, New York, 1948.

[17] L. F. Ho and D. L. Russell, Admissible Input Elements for Systems in Hilbert Space and a Carleson Measure Criterion, SIAM J. Control Optim., 21(4), 614-640.

[18] G. Lebeau and L. Robbiano, Contrôle exact de l'équation de la chaleur, Comm. Partial Differential Equations, 20 (1995), pp. 335-356. 
[19] V. Komornik and P. Loreti, Fourier series in control theory, Springer, 2005.

[20] M. Léautaud, Uniform controllability of scalar conservation laws in the vanishing viscosity limit, SIAM, J. Control Optim. 50, 1661-1699, 2012.

[21] Lissy, P., A link between the cost of fast controls for the 1-D heat equation and the uniform controllability of a 1-D transport-diffusion equation. C. R. Math. Acad. Sci. Paris 350 (2012), no. 11-12, 591-595.

[22] Lissy, P., An application of a conjecture due to Ervedoza and Zuazua concerning the observability of the heat equation in small time to a conjecture due to Coron and Guerrero concerning the uniform controllability of a convection-diffusion equation in the vanishing viscosity limit, Systems and Control Letters 69 (2014), 98-102.

[23] Lissy, P., On the Cost of Fast Controls for Some Families of Dispersive or Parabolic Equations in One Space Dimension SIAM J. Control Optim., 52(4), 2651-2676.

[24] Lissy, P., Explicit lower bounds for the cost of fast controls for some 1-D parabolic or dispersive equations, and a new lower bound concerning the uniform controllability of the 1-D transport-diffusion equation, J. Differential Equations 259 (2015), no. 10, 5331-5352.

[25] L. Lorch and M. E. Muldoon, Monotonic sequences related to zeros of Bessel functions, Numer. Algor. (2008) 49:221-233.

[26] J.-L. Lions and E. Magenes, Non-homogeneous boundary value problems and applications. Springer Berlin, 1972 .

[27] F. W. J. Olver, Asymptotics and special functions, New York, Academic Press, 1974.

[28] Koosis P., The logarithmic integral I \& II, Cambridge Studies in Advanced Mathematics 12 (1988) \& Cambridge Studies in Advanced Mathematics 21 (1992), Cambridge University Press, Cambridge.

[29] G. Metafune and D. Pallara, Trace Formulas for Some Singular Differential Operators and Applications, Math. Nachr. 211 (2000), 127-157.

[30] Walter Rudin. Real and complex analysis. McGraw-Hill Book Co., New York, 1966.

[31] H. Tanabe, Equations of evolution, Pitman, London, 1979; English transl., Iwanami, Tokyo, 1975.

[32] G. Tenenbaum and M. Tucsnak, New blow-up rates for fast controls of Schrodinger and heat equations. J. Differential Equations 243 (2007), no. 1, 70-100.

[33] G. N. Watson, A treatise on the theory of Bessel functions, Cambridge University Press, Cambridge, England, 1958 . 